\title{
Transverse charge and magnetization densities in holographic QCD
}

\author{
Dipankar Chakrabarti ${ }^{\mathrm{a}}$, Chandan Mondal \\ Department of Physics, Indian Institute of Technology Kanpur, Kanpur 208016, India
}

Received: 2 May 2014 / Accepted: 1 July 2014 / Published online: 18 July 2014

(C) The Author(s) 2014. This article is published with open access at Springerlink.com

\begin{abstract}
We present a study of the flavor structures of the transverse charge and anomalous magnetization densities for both unpolarized and transversely polarized nucleons. We consider two different models for the electromagnetic form factors in holographic QCD. The flavor form factors are obtained by decomposing the Dirac and Pauli form factors for the nucleons using the charge and isospin symmetry. The results are compared with two standard phenomenological parametrizations.
\end{abstract}

\section{Introduction}

Form factors of the nucleons provide us with crucial information about the internal structure of the nucleons and have been measured in many experiments. The charge and magnetization densities in the transverse plane are defined as the Fourier transforms of the electromagnetic form factors. The transverse densities are also intimately related to the generalized parton distributions (GPDs) with zero skewness. The contributions of an individual quark to the nucleon charge and magnetization densities are obtained from the flavor decompositions of the transverse densities. The transverse densities corresponding to the individual quarks are given by the Fourier transforms of the GPDs in the transverse coordinate or impact parameter space [1,2]. The form factor involves initial and final states with different momenta, and three dimensional Fourier transforms cannot be interpreted as densities, whereas the transverse densities defined at fixed light-front time are free from this difficulty and have a proper density interpretation [3-5].

Recently, AdS/QCD has emerged as one of the most promising techniques to unravel the structure of mesons and nucleons. The AdS/CFT conjecture [6] relates a gravity theory in $A d S_{d+1}$ to a conformal theory at the $d$ dimensional boundary. There are many applications of AdS/CFT to inves-

a e-mail: dipankar@iitk.ac.in tigate the QCD phenomena [7-12]. A boundary condition in the fifth dimension $z$ in $A d S_{5}$ breaks the conformal invariance and allows for a QCD mass scale and confinement. In the hard-wall model, an IR cutoff is set at $z_{0}=1 / \Lambda_{\mathrm{QCD}}$, while in soft-wall model, a confining potential in $z$ is introduced. There is an exact correspondence between the holographic variable $z$ and the light-front transverse variable $\zeta$ which measures the separation of the quark and gluonic constituents in the hadron [13-15]. The AdS/QCD for the baryon has been developed by several groups [13-21]. Though it gives only a semiclassical approximation of QCD, so far this method has been successfully applied to describe many hadron properties e.g., hadron mass spectrum, parton distribution functions, GPDs, meson and nucleon form factors, structure functions etc. [18,22-33]. AdS/QCD wave functions are used to predict the experimental data for $\rho$ meson electroproduction [34]. AdS/QCD has also been successfully applied in the meson sector to predict the branching ratio for decays of $\bar{B}^{0}$ and $\overline{B_{s}^{0}}$ into $\rho$ mesons [35], isospin asymmetry and branching ratio for the $B \rightarrow K^{*} \gamma$ decays [36], transition form factors $[37,38]$, etc. There are many other applications in the baryon sector; e.g., semi-empirical hadronic momentum density distributions in the transverse plane have been calculated in [39], in [40], the form factor of spin $3 / 2$ baryons ( $\Delta$ resonance) and also the transition form factor between $\Delta$ and nucleon have been studied, and an AdS/QCD model has been proposed to study the baryon spectrum at finite temperature [41], etc.

The flavor decompositions of the nucleon form factors in a light-front quark model with SU(6) spin-flavor symmetry have been studied in detail in [32] and shown to agree with experimental data. It is interesting and instructive to study the transverse densities and their flavor decomposition in holographic QCD. There are two different holographic QCD models for nucleon form factors developed by Abidin and Carlson [18] and Brodsky and Teramond [27]. Here, we present a detailed analysis of the transverse densities in the two models. 
Model independent transverse charge densities for nucleons have been studied in [42] whereas the charge densities in the transverse plane for a transversely polarized nucleon are shown in [43,44]. In [45], the long range behavior of the unpolarized quark transverse charge density of the nucleons has been studied. Transverse charge and magnetization densities in the nucleon's chiral periphery (i.e., at a distance $\left.b=\mathcal{O}\left(1 / m_{\pi}\right)\right)$ using methods of dispersion analysis and chiral effective field theory have been analyzed in [46]. The transverse densities for the quarks are studied in a chiral quark-soliton model in [47]. Using a LaguerreGaussian expansion, Kelly [48] proposed a parametrization of the nucleon Sachs form factors in terms of charge and magnetization densities. A study of the flavor dependence of the transverse densities in a GPD model has been reported in [49].

In [28-30], the nucleon transverse charge and magnetization densities have been evaluated in the model developed in [18]. In this work, we show the flavor decompositions of the transverse densities of the nucleons in two different models in the framework of AdS/QCD and compare with the two global parametrizations of Kelly [50] and Bradford et al. [51]. By decomposing the nucleon form factors $F_{1}$ and $F_{2}$ using the charge and isospin symmetry, we obtain the flavor form factors $F_{1}^{q}$ and $F_{2}^{q}$ for the quarks. The Fourier transforms of these electromagnetic form factors give the charge and magnetization densities in the transverse plane.

The paper is organized as follows. A brief description of the form factors in AdS/QCD has been has given in Sect. 2 . In Sect. 3, the charge and magnetization densities for both unpolarized and transversely polarized nucleons have been studied. The individual flavor contributions are also studied in this section. Then we provide a brief summary in Sect. 4.

\section{Nucleon and flavor form factors in AdS/QCD}

Here we consider the soft-wall model of AdS/QCD, where in place of a sharp cutoff in $\mathrm{z}$, one introduces a potential. The action in soft model is written as [27]

$$
\begin{aligned}
S= & \int \mathrm{d}^{4} x \mathrm{~d} z \sqrt{g}\left(\frac{i}{2} \bar{\Psi} e_{A}^{M} \Gamma^{A} D_{M} \Psi-\frac{i}{2}\left(D_{M} \bar{\Psi}\right) e_{A}^{M} \Gamma^{A} \Psi\right. \\
& -\mu \bar{\Psi} \Psi-V(z) \bar{\Psi} \Psi),
\end{aligned}
$$

where $e_{A}^{M}=(z / R) \delta_{A}^{M}$ is the inverse vielbein and $V(z)$ is the confining potential which breaks the conformal invariance and $R$ is the AdS radius. The covariant derivative is $D_{M}=$ $\partial_{M}-\frac{i}{2} \omega_{M}^{A B} \Sigma_{A B}$ where $\omega_{M}^{A B}=\left(\eta^{A z} \delta_{M}^{B}-\eta^{B z} \delta_{M}^{A}\right) / z$ and $\Sigma_{A B}=\frac{i}{4}\left[\Gamma_{A}, \Gamma_{B}\right]$.

The Dirac equation in AdS derived from the above action is given by $i\left(z \eta^{M N} \Gamma_{M} \partial_{N}+\frac{d}{2} \Gamma_{z}\right) \Psi-\mu R \Psi-R V(z) \Psi=0$.

Here $z$ is identified as the light-front transverse impact variable $\zeta$ which gives the separation of the quark and gluonic constituents in the hadron, it is then possible to extract the light-front wave functions for the hadron. In $d=4$ dimensions, $\Gamma_{A}=\left\{\gamma_{\mu},-i \gamma_{5}\right\}$. To map with the light-front wave equation, we identify $z \rightarrow \zeta$, where $\zeta$ is the light-front transverse variable, and substitute $\Psi(x, \zeta)=e^{-i P \cdot x} \zeta^{2} \psi(\zeta) u(P)$ in Eq. (2) and set $|\mu R|=v+1 / 2$ where $v$ is related with the orbital angular momentum by $v=L+1$. For a linear confining potential $U(\zeta)=(R / \zeta) V(\zeta)=\kappa^{2} \zeta$, we get the light-front wave equation for the baryon in $2 \times 2$ spinor representation as

$$
\begin{aligned}
& \left(-\frac{\mathrm{d}^{2}}{\mathrm{~d} \zeta^{2}}-\frac{1-4 \nu^{2}}{4 \zeta^{2}}+\kappa^{4} \zeta^{2}+2(\nu+1) \kappa^{2}\right) \psi_{+}(\zeta) \\
& =\mathcal{M}^{2} \psi_{+}(\zeta) \\
& \left(-\frac{\mathrm{d}^{2}}{\mathrm{~d} \zeta^{2}}-\frac{1-4(\nu+1)^{2}}{4 \zeta^{2}}+\kappa^{4} \zeta^{2}+2 \nu \kappa^{2}\right) \psi_{-}(\zeta) \\
& =\mathcal{M}^{2} \psi_{-}(\zeta) .
\end{aligned}
$$

In the case of mesons, a similar potential $\kappa^{4} \zeta^{2}$ appears in the Klein-Gordon equation which can be generated by introducing a dilaton background $\phi=e^{ \pm \kappa^{2} z^{2}}$ in the AdS space which breaks the conformal invariance. But in the case of a baryon, the dilaton can be scaled out by a field redefinition [27]. So, the confining potential for baryons cannot be produced by a dilaton and is put in by hand in the soft-wall model. The form of the confining potential $\left(\kappa^{4} \zeta^{2}\right)$ is unique for both the meson and the baryon sectors [52]. The solutions of the above equations are

$$
\begin{aligned}
& \psi_{+}(z) \sim \zeta^{\nu+1 / 2} e^{-\kappa^{2} \zeta^{2} / 2} L_{n}^{\nu}\left(\kappa^{2} \zeta^{2}\right), \\
& \psi_{-}(z) \sim \zeta^{\nu+3 / 2} e^{-\kappa^{2} \zeta^{2} / 2} L_{n}^{\nu+1}\left(\kappa^{2} \zeta^{2}\right) .
\end{aligned}
$$

\section{Model I}

By model I, we refer to the AdS/QCD model for the form factors proposed by Brodsky and Teramond [27]. The SU(6) spin-flavor symmetric quark model is constructed in the AdS/QCD by weighing the different Fock-state components by the charges and spin projections of the partons as dictated by the symmetry. In the light-front quark model the Dirac and Pauli form factors are described by the spin-nonflip and spin-flip matrix elements of the electromagnetic current $J^{+}=e_{q} \bar{\psi} \gamma^{+} \psi[54]$.

The Dirac form factors for the nucleons are obtained in this model as

$$
\begin{aligned}
& F_{1}^{p}\left(Q^{2}\right)=R^{4} \int \frac{\mathrm{d} z}{z^{4}} V\left(Q^{2}, z\right) \psi_{+}^{2}(z) \\
& F_{1}^{n}\left(Q^{2}\right)=-\frac{1}{3} R^{4} \int \frac{\mathrm{d} z}{z^{4}} V\left(q^{2}, z\right)\left(\psi_{+}^{2}(z)-\psi_{-}^{2}(z)\right),
\end{aligned}
$$



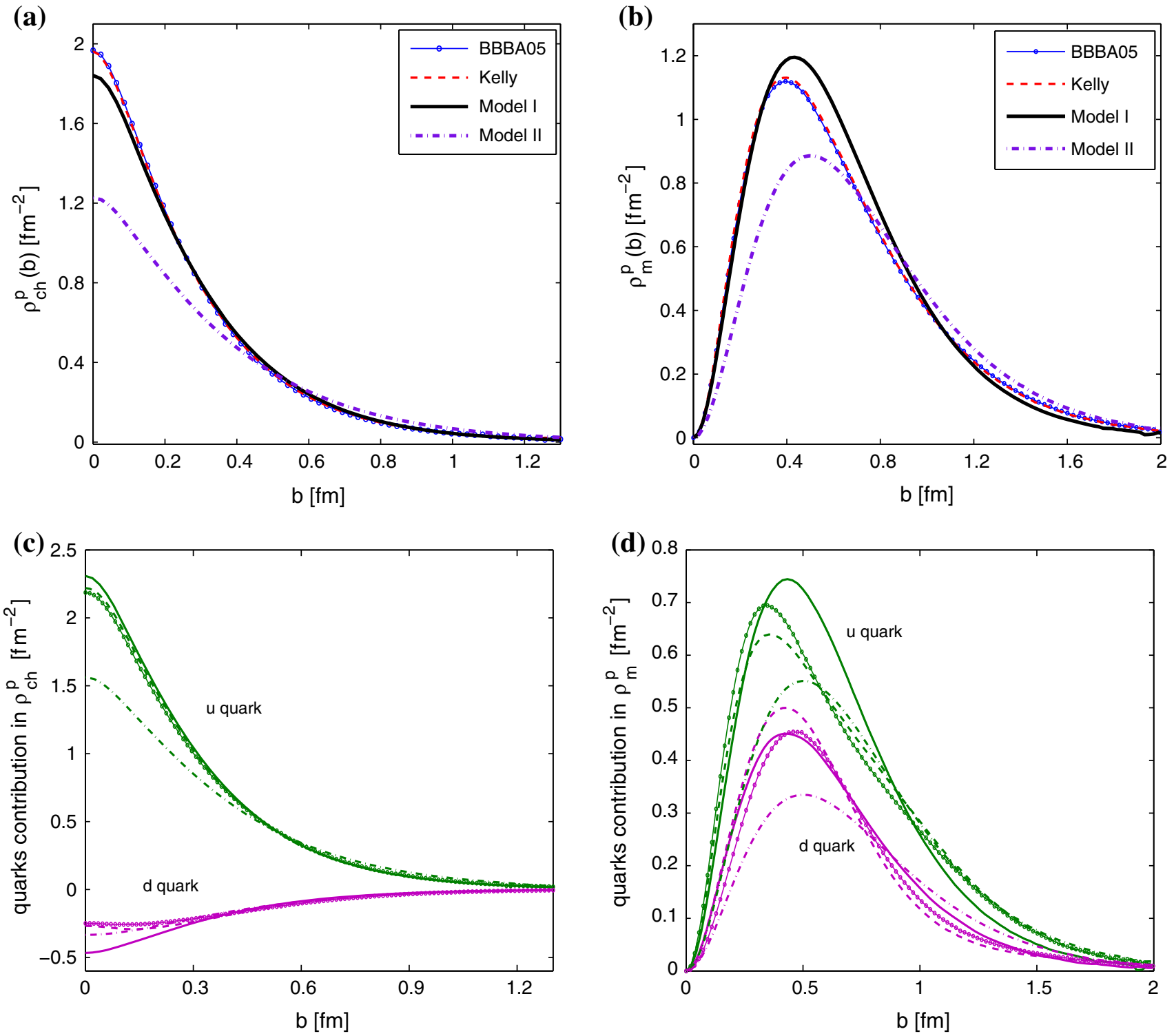

Fig. 1 Plots of flavor dependent transverse charge and anomalous magnetization densities for proton. $\mathbf{a}$ and $\mathbf{b}$ Represent $\rho_{c h}$ and $\rho_{m}$ for the proton. $\mathbf{c}$ and $\mathbf{d}$ Represent the contributions from different flavors. A dashed line represents the parametrization of Kelly [50], and the line

and the Pauli form factor is written as

$F_{2}^{p / n}\left(Q^{2}\right) \sim \int \frac{\mathrm{d} z}{z^{3}} \psi_{+}(z) V\left(Q^{2}, z\right) \psi_{-}(z)$.

The form factors are normalized to $F_{1}^{p}(0)=1, F_{1}^{n}(0)=0$, and $F_{2}^{p / n}(0)=\kappa_{p / n}$, where $\kappa_{p / n}$ is the anomalous magnetic moments for the proton/neutron. Note that the Pauli form factor is not mapped properly in this model. In the light-front quark model, it is defined as the spin-flip matrix element of the $J^{+}$current but the AdS action cannot produce this term and the Pauli form factor is put in for phenomenological purposes. The twist-3 nucleon wave functions in the softwall model are

with circles represents the parametrization of Bradford et al. [51]; the solid line is for Model I and the dot-dashed line is for Model II. In c and $\mathbf{d} u$ and $d$ quark contributions are plotted in different colors

$\psi_{+}(z)=\frac{\sqrt{2} \kappa^{2}}{R^{2}} z^{7 / 2} e^{-\kappa^{2} z^{2} / 2}$,
$\psi_{-}(z)=\frac{\kappa^{3}}{R^{2}} z^{9 / 2} e^{-\kappa^{2} z^{2} / 2}$.

The bulk-to-boundary propagator is given by

$V\left(Q^{2}, z\right)=\Gamma\left(1+\frac{Q^{2}}{4 \kappa^{2}}\right) U\left(\frac{Q^{2}}{4 \kappa^{2}}, 0, \kappa^{2} z^{2}\right)$,

where $U(a, b, z)$ is the Tricomi confluent hypergeometric function. The bulk-to-boundary propagator, Eq. (12), can be written in a simple integral form [27,53], 

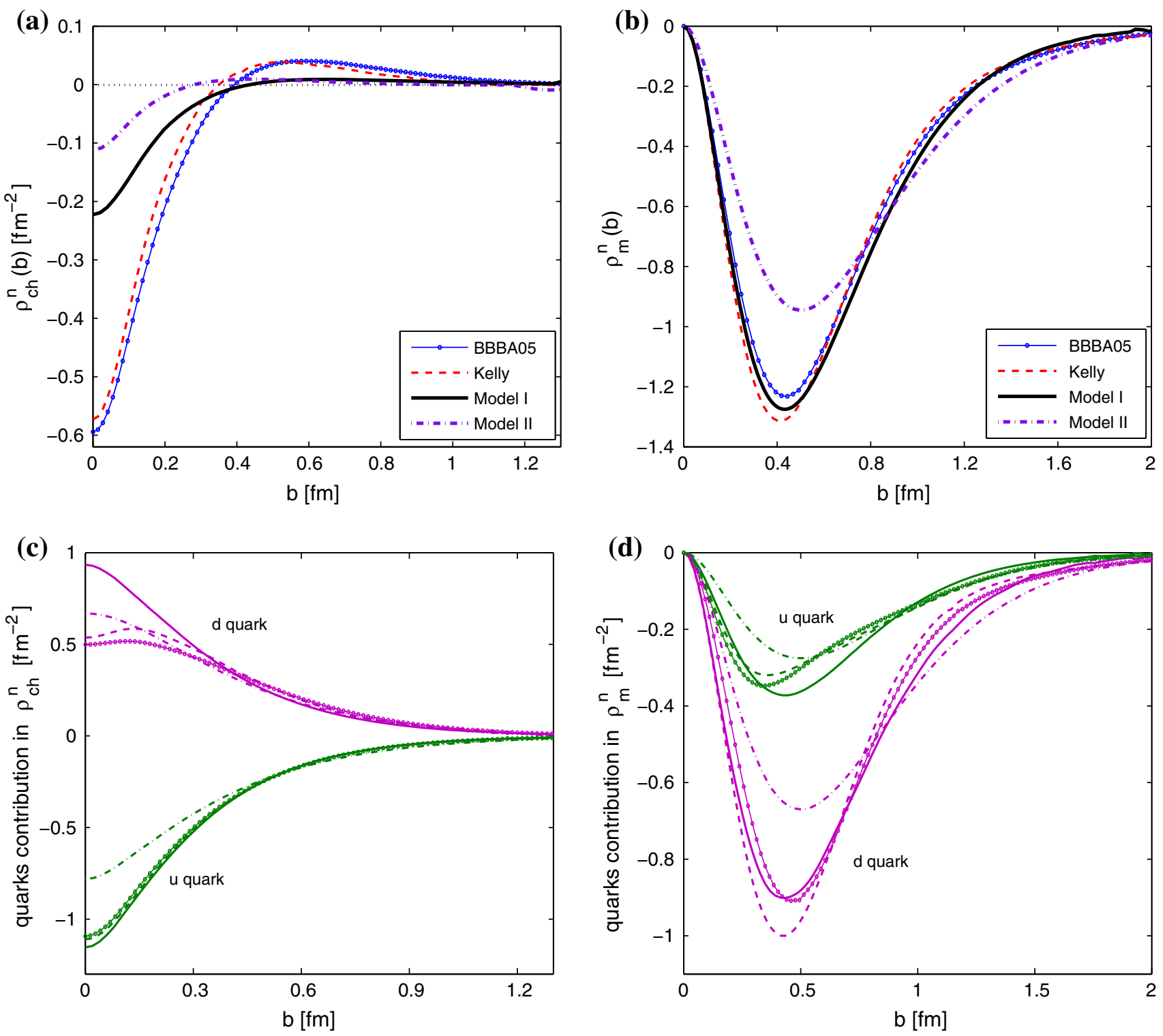

Fig. 2 Plots of flavor dependent transverse charge and anomalous magnetization densities for the neutron. $\mathbf{a}$ and $\mathbf{b}$ Represent $\rho_{c h}$ and $\rho_{m}$ for the neutron. $\mathbf{c}$ and $\mathbf{d}$ Represent the contributions from different flavors. A dashed line represents the parametrization of Kelly [50], and the line

$V\left(Q^{2}, z\right)=\kappa^{2} z^{2} \int_{0}^{1} \frac{\mathrm{d} x}{(1-x)^{2}} x^{Q^{2} /\left(4 \kappa^{2}\right)} e^{-\kappa^{2} z^{2} x /(1-x)}$.

We refer to the formulas for the form factors given in Eqs. (7), (8), and (9) as Model I. It has been shown [31,32] that the form factors for the nucleons agree with the experimental data for $\kappa=0.4066 \mathrm{GeV}$.

\section{Model II}

The other model of the form factors was formulated by Abidin and Carlson [18]. Since the action defined in Eq. (1) cannot produce the spin-flip (Pauli) form factors, they intro-

with circles represents the parametrization of Bradford et al. [51]; the solid line is for Model I and the dot-dashed line is for Model II. In c and $\mathbf{d} u$ and $d$ quark contributions are plotted in different colors

duced an additional gauge invariant non-minimal coupling. This additional term also gives an anomalous contribution to the Dirac form factor. In this model the form factors are given by [18]

$F_{1}^{p}\left(Q^{2}\right)=C_{1}\left(Q^{2}\right)+\eta_{p} C_{2}\left(Q^{2}\right)$,

$F_{1}^{n}\left(Q^{2}\right)=\eta_{n} C_{2}\left(Q^{2}\right)$,

$F_{2}^{p}\left(Q^{2}\right)=\eta_{p} C_{3}\left(Q^{2}\right)$,

$F_{2}^{n}\left(Q^{2}\right)=\eta_{n} C_{3}\left(Q^{2}\right)$.

Here

$C_{1}\left(Q^{2}\right)=\frac{a+6}{(a+1)(a+2)(a+3)}$, 


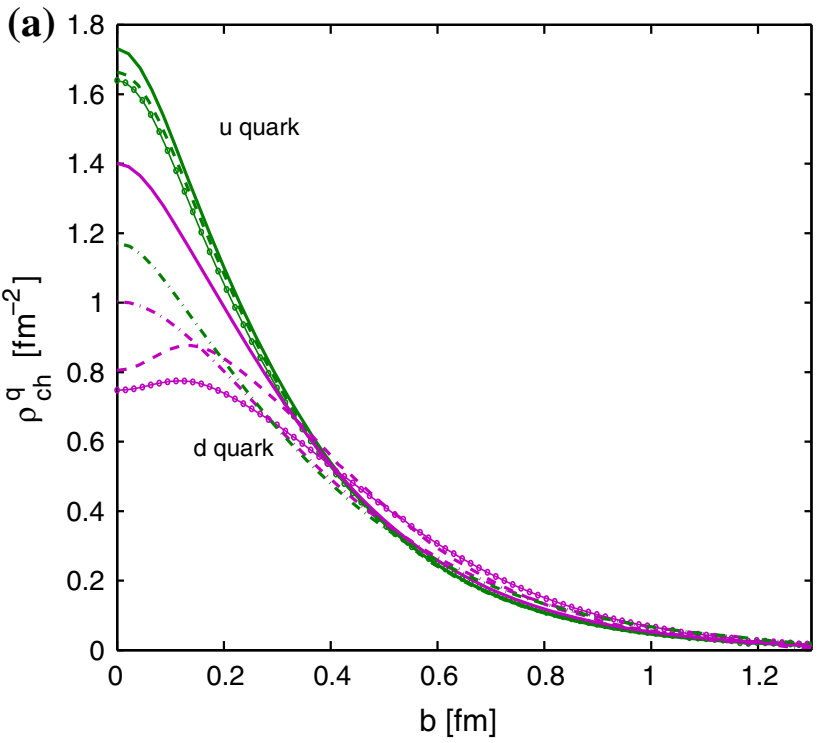

Fig. 3 Plots of quarks transverse charge and anomalous magnetization densities. a Represents $\rho_{c h}^{q}$ and $\mathbf{b}$ represents $\rho_{m}^{q}$. A dashed line represents the parametrization of Kelly [50], and a line with circles represents

$$
\begin{aligned}
& C_{2}\left(Q^{2}\right)=\frac{2 a(2 a-1)}{(a+1)(a+2)(a+3)(a+4)}, \\
& C_{3}\left(Q^{2}\right)=\frac{48}{(a+1)(a+2)(a+3)},
\end{aligned}
$$

where $a=Q^{2} /\left(4 \kappa^{2}\right)$. The value of $\kappa$ is fixed by a simultaneous fit to the proton and the rho meson mass and the best fit gives the value $\kappa=0.350 \mathrm{GeV}$. The other parameters are determined from the normalization conditions of the Pauli form factor at $Q^{2}=0$ and are given by $\eta_{p}=0.224$ and $\eta_{n}=-0.239$ [18]. We refer to the form factors given by Eqs. (14)-(17) as Model II.

The Pauli form factors in these two models are identical; the main difference is in the Dirac form factor. In Model II, there is an additional contribution to the Dirac form factor from the non-minimal coupling term. It should be mentioned here that the Pauli form factors in the AdS/QCD models are mainly of phenomenological origin. Since the action in Eq. (1) cannot produce the spin-flip term, in Model II, a nonminimal coupling term is added to generate the Pauli form factors. This additional term gives a contribution to the Dirac form factors also. The major difference between these two models is that in Model I, the anomalous contributions to the Dirac form factors are not considered. The additional contribution from the non-minimal coupling to the Dirac form factor corresponds to a higher twist and is not included in Model I, while they are included in Model II.

Under the charge and isospin symmetry it is straightforward to write down the flavor decompositions of the nucleon form factors as

$F_{i}^{u}=2 F_{i}^{p}+F_{i}^{n}$ and $F_{i}^{d}=F_{i}^{p}+2 F_{i}^{n}(i=1,2)$,

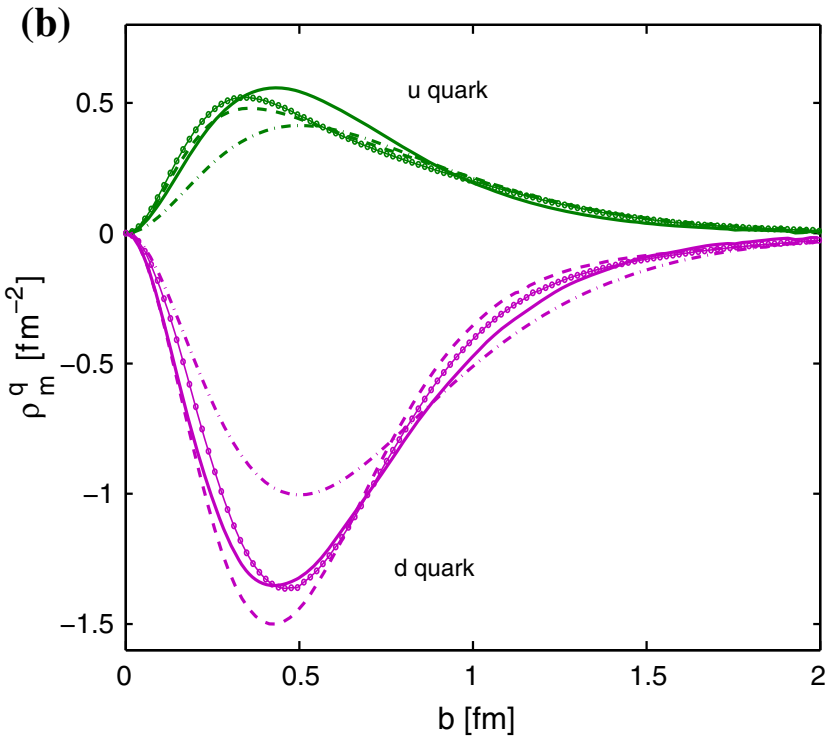

the parametrization of Bradford et al. [51]; the solid line is for Model I and the dot-dashed line is for Model II. The densities for the $u$ and $d$ quark are plotted in different colors

with the normalizations $F_{1}^{u}(0)=2, F_{2}^{u}(0)=\kappa_{u}$ and $F_{1}^{d}(0)$ $=1, F_{2}^{d}(0)=\kappa_{d}$, where the anomalous magnetic moments for the up and down quarks are $\kappa_{u}=2 \kappa_{p}+\kappa_{n}=1.673$ and $\kappa_{d}=\kappa_{p}+2 \kappa_{n}=-2.033$. It was shown in [55] that, though the ratio of Pauli and Dirac form factors for the proton is $F_{2}^{p} / F_{1}^{p} \propto 1 / Q^{2}$, the $Q^{2}$ dependence is almost constant for the ratio of the quark form factors $F_{2} / F_{1}$ for both $u$ and $d$.

\section{Transverse charge and magnetization densities}

The transverse charge density inside the nucleons is given by

$$
\begin{aligned}
\rho_{c h}(b) & =\int \frac{\mathrm{d}^{2} q_{\perp}}{(2 \pi)^{2}} F_{1}\left(q^{2}\right) e^{i q_{\perp} \cdot b_{\perp}} \\
& =\int_{0}^{\infty} \frac{\mathrm{d} Q}{2 \pi} Q J_{0}(Q b) F_{1}\left(Q^{2}\right),
\end{aligned}
$$

where $b$ represents the impact parameter and $J_{0}$ is the cylindrical Bessel function of order zero. A similar formula for the charge density for the flavor $\rho_{f c h}^{q}(b)$ can be written with $F_{1}$ replaced by $F_{1}^{q}$. One can define the magnetization density in a similar fashion to have the formula

$$
\begin{aligned}
\widetilde{\rho}_{M}(b) & =\int \frac{\mathrm{d}^{2} q_{\perp}}{(2 \pi)^{2}} F_{2}\left(q^{2}\right) e^{i q_{\perp} \cdot b_{\perp}} \\
& =\int_{0}^{\infty} \frac{\mathrm{d} Q}{2 \pi} Q J_{0}(Q b) F_{2}\left(Q^{2}\right),
\end{aligned}
$$



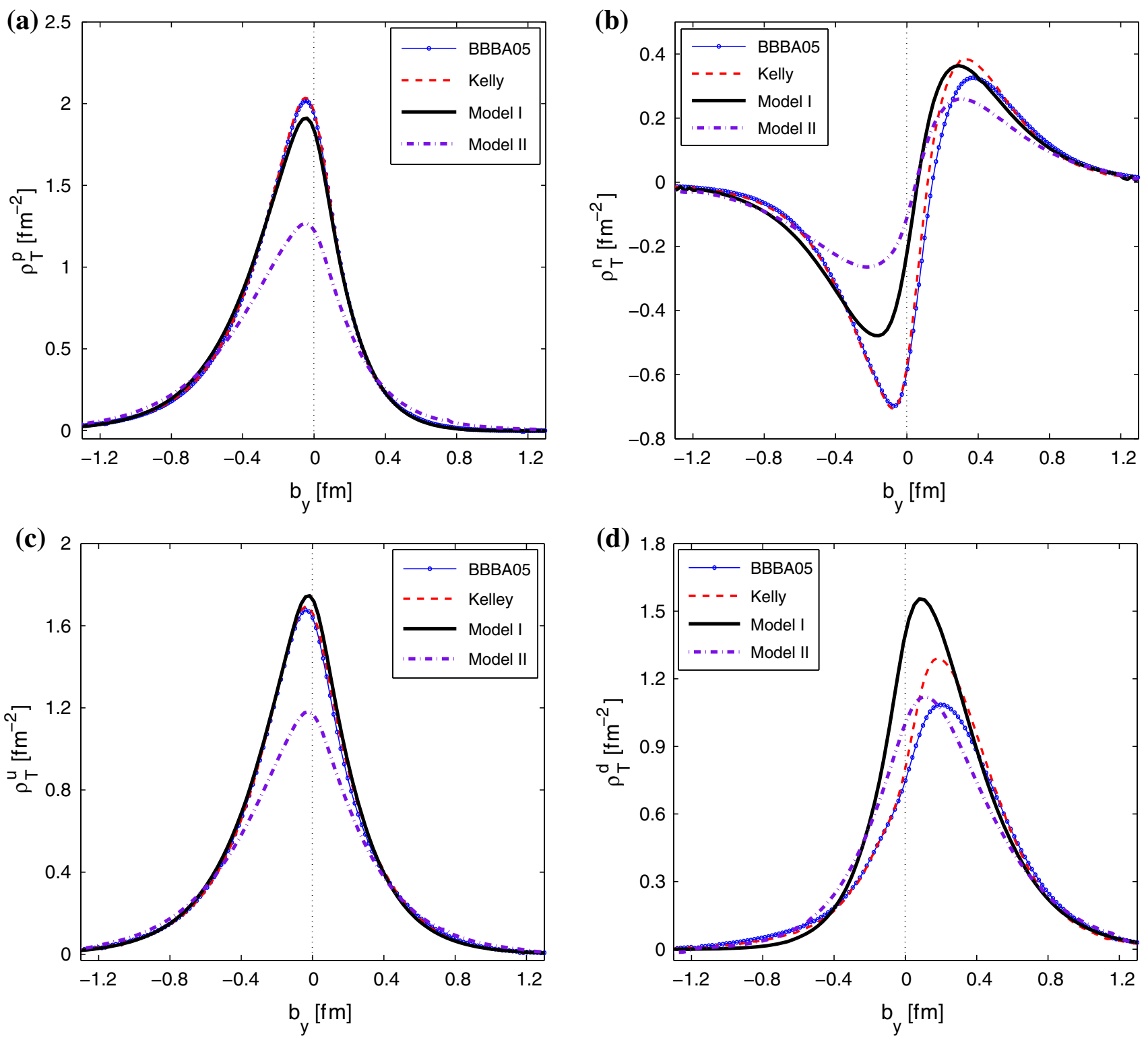

Fig. 4 The charge densities for the transversely polarized a proton, b neutron, and $\mathbf{c}$ up and $\mathbf{d}$ down quark charge densities for the transversely polarized nucleon. A dashed line represents the parametrization

whereas

$\rho_{m}(b)=-b \frac{\partial \widetilde{\rho}_{M}(b)}{\partial b}=b \int_{0}^{\infty} \frac{\mathrm{d} Q}{2 \pi} Q^{2} J_{1}(Q b) F_{2}\left(Q^{2}\right)$

has the interpretation of an anomalous magnetization density [4]. Since these quantities are not directly measured in experiments, actual experimental data are not available. In [5], an approximate estimation of the proton charge and magnetization densities has been done from experimental form factor data. To get an insight into the contributions of the different quark flavors, we evaluate the charge and anomalous magnetization densities for the up and down quarks.

of Kelly [50], and a line with circles represents the parametrization of Bradford et al. [51]; the solid line is for Model I and the dot-dashed line is for Model II

We can define the decompositions of the transverse charge and magnetization densities for nucleons in a similar way as electromagnetic form factors [55]. The charge density decompositions in terms of two flavors can be written as

$\rho_{c h}^{p}=e_{u} \rho_{f c h}^{u}+e_{d} \rho_{f c h}^{d}$,

$\rho_{c h}^{n}=e_{u} \rho_{f c h}^{d}+e_{d} \rho_{f c h}^{u}$,

where $e_{u}$ and $e_{d}$ are the charge of the $u$ and $d$ quarks, respectively. We should remember that due to the charge and isospin symmetry, the $u, d$ quark densities in the proton are the same as the $d, u$ densities in the neutron, as shown in [42], 

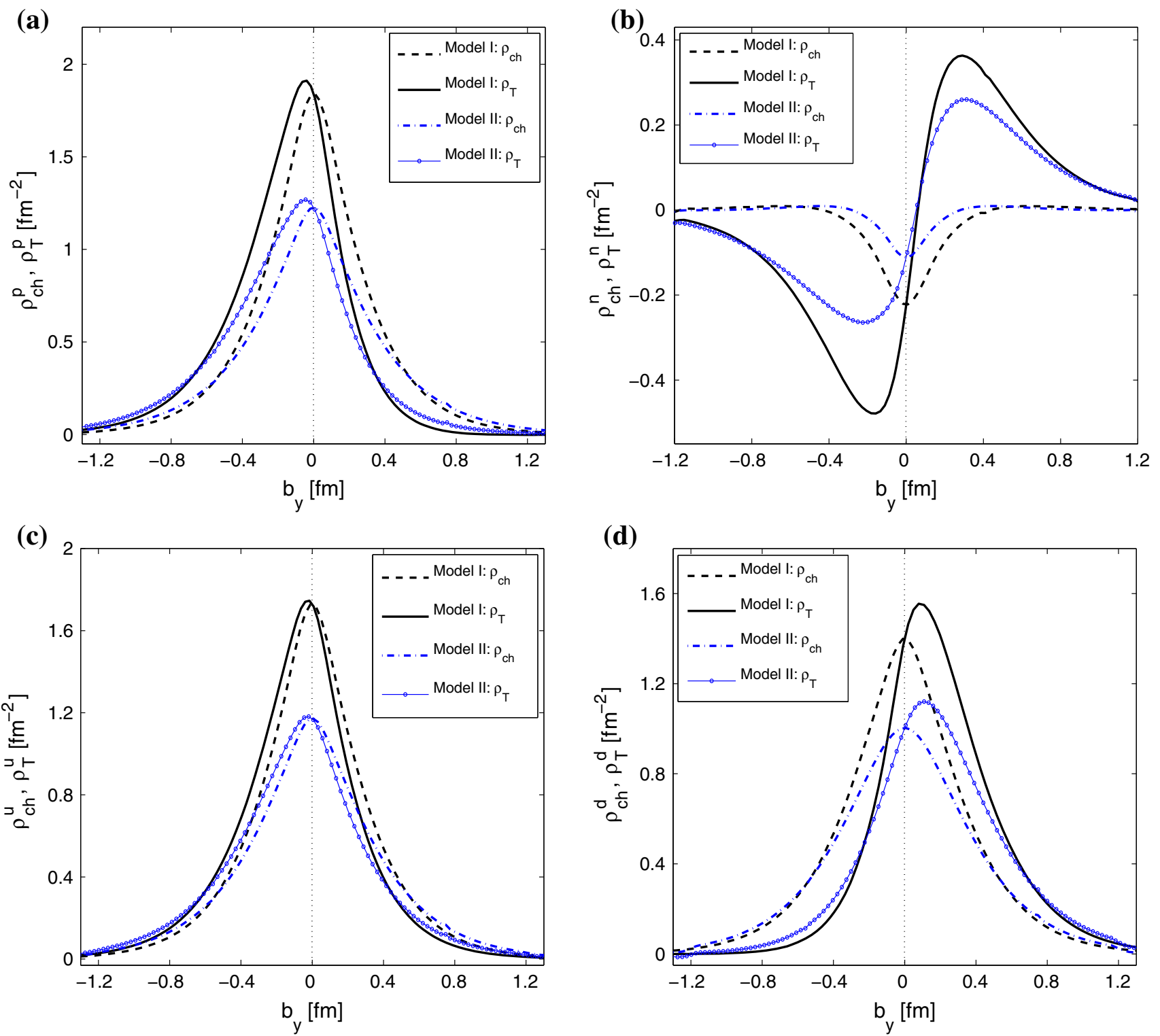

Fig. 5 The charge densities for the transversely polarized a proton, $\mathbf{b}$ neutron, and $\mathbf{c}$ up and $\mathbf{d}$ down quark charge densities for the transversely polarized nucleon. The unpolarized charge densities are shown by the dashed line

$\rho_{c h}^{u}(b)=\rho_{c h}^{p}+\frac{\rho_{c h}^{n}}{2}=\frac{\rho_{f c h}^{u}}{2}$,

$\rho_{c h}^{d}(b)=\rho_{c h}^{p}+2 \rho_{c h}^{n}=\rho_{f c h}^{d}$,

where $\rho_{c h}^{q}(b)$ is the charge density of each quark and $\rho_{f c h}^{q}$ is the charge density for each flavor. We can also do similar decompositions to Eqs. (25) and (26) for $\rho_{m}$.

We are not aware of any experimental data on transverse densities. Kelly [50] and Bradford et al. [51] proposed two different phenomenological parameterizations of the nucleon form factor data. Here we calculate the transverse charge and magnetization densities from these two parameterizations and compare with AdS/QCD predictions. Miller [42] also used these parameterizations to evaluate the transverse charge densities of the nucleons. In Fig. 1a, b we show the charge and anomalous magnetization densities for the proton. The plots suggest that Model I agrees with the phenomenological parametrizations much better than Model II. The flavors contributions coming to proton densities from $e_{u / d} \rho_{f c h}^{u / d}$ and $e_{u / d} \rho_{f m}^{u / d}$ are shown in Fig. 1c,d. Similarly the charge and anomalous magnetization densities for neutron and the flavors contributions $e_{d / u} \rho_{f c h}^{u / d}$ and $e_{d / u} \rho_{f m}^{u / d}$ are shown in Fig. 2. At small $b$, both holographic models fail to reproduce the neutron charge density. Model I reproduces the neutron magnetization density, while Model II again fails to agree as shown in Fig. 2b. Model I results for the $u$ quark contributions to the charge density for both proton and neutron are in excellent agreement with the two different global 

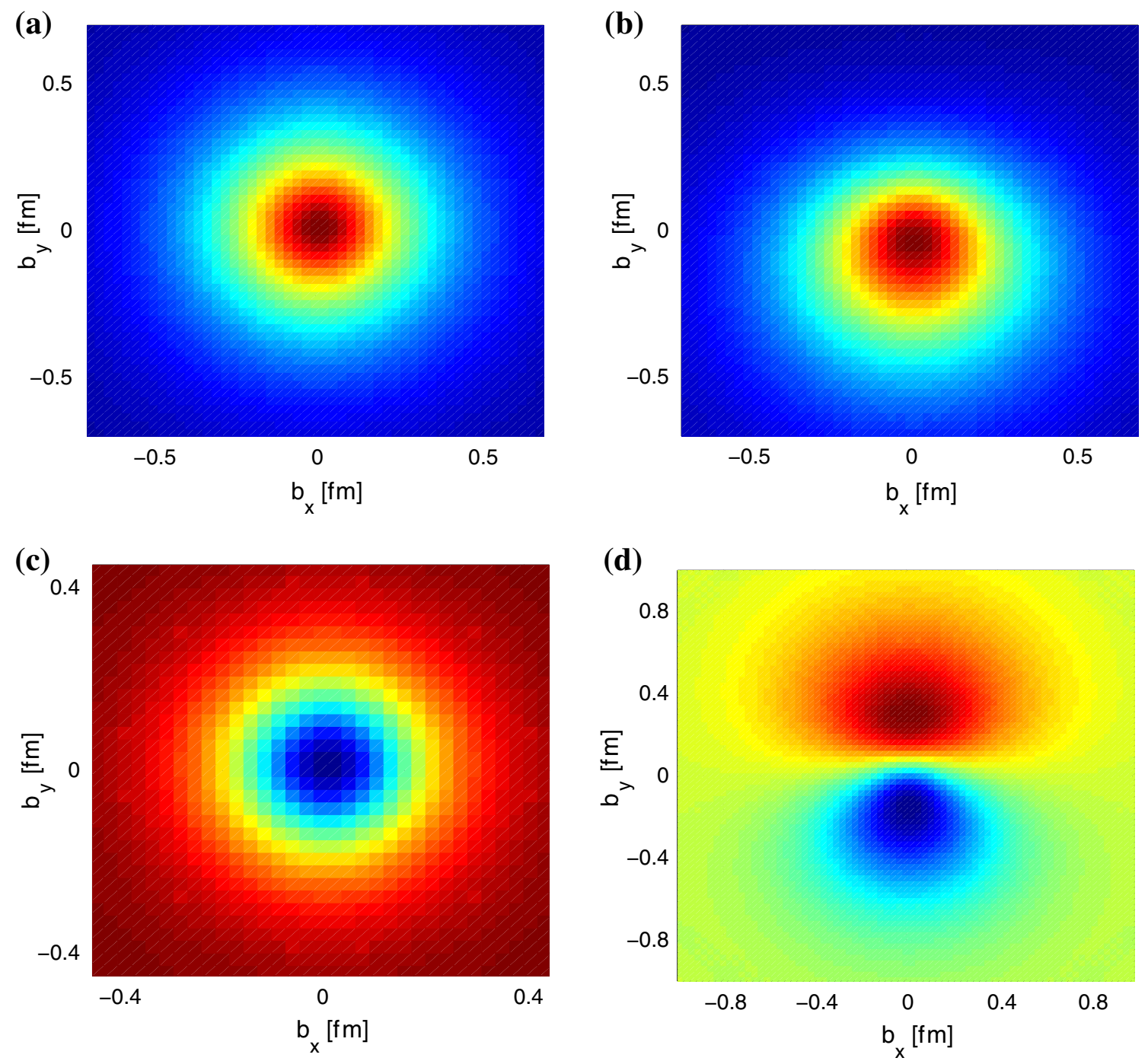

Fig. 6 The charge densities in the transverse plane for the a unpolarized proton, $\mathbf{b}$ transversely polarized proton, and $\mathbf{c}$ unpolarized neutron and $\mathbf{d}$ transversely polarized neutron. The transverse polarization is along the $x$ direction

parametrizations of Kelly [50] and Bradford et al. [51]. The $d$ quark contributions deviate from these two fits. It is not very surprising as it has been already shown [32] for Model I that the Dirac form factor for the $d$ quark itself does not agree well with the experiment results. In the case of an anomalous magnetization both the quarks contributions in the proton and the neutron agree quite well with the fits. The charge density for the neutron (Fig. 2a) shows a negatively charged core surrounded by a ring of positive charge density (note that $b=0$ corresponds to the center of the nucleon). In the proton charge density the contribution of the up quark is large enough compared to the down quark, but for the neutron both contributions from $u$ and $d$ quark are comparable. For the anomalous magnetization density of the neutron, the $d$ quark contribution is quite high compared to the $u$ quark. In Fig. 3a,b we show the individual quark's charge and anomalous magnetization densities. The charge density for $d$ quark in Model I deviates from the fits but is in excellent agreement for the $u$ quark. But again, as said before, a deviation for the $d$ quark in Model I is expected. The anomalous magnetization densities in both $u$ and $d$ quarks in Model I match very well with the fits. It is positive for the $u$ quark but negative and larger for the $d$ quark. The Model II result for the anomalous magnetization density of the $d$ quark does not match so well with the phenomenological fits as Model I.

For a transversely polarized nucleon, the charge density is given by [44]

$\rho_{T}(b)=\rho_{c h}-\sin \left(\phi_{b}-\phi_{s}\right) \frac{1}{2 M b} \rho_{m}$,

where $M$ is the mass of the nucleon and the transverse polarization of the nucleon is given by $S_{\perp}=\left(\cos \phi_{s} \hat{x}+\sin \phi_{s} \hat{y}\right)$ and the transverse impact parameter $b_{\perp}=b\left(\cos \phi_{b} \hat{x}+\right.$ $\left.\sin \phi_{b} \hat{y}\right)$. Without loss of generality, the polarization of the 

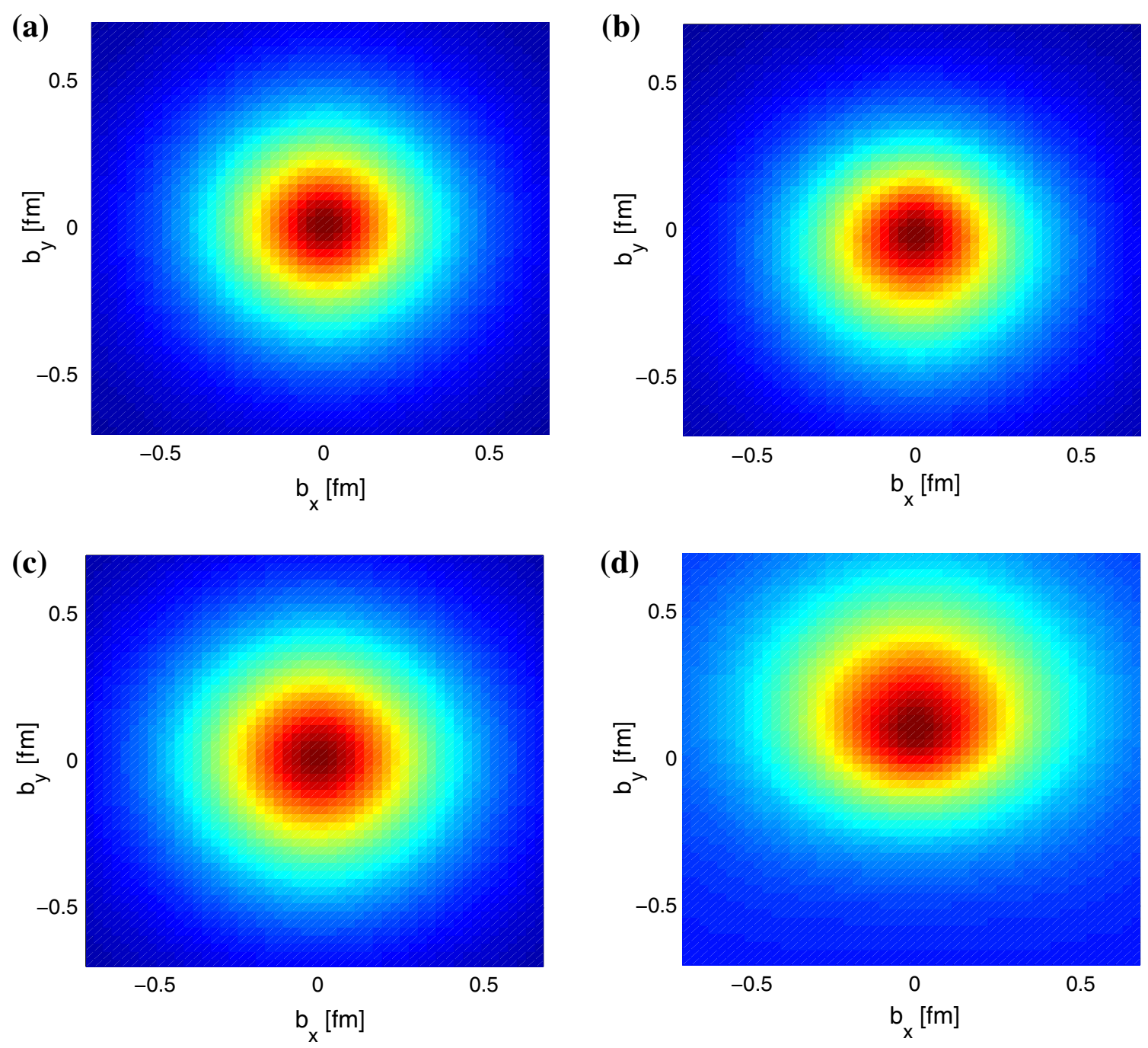

Fig. 7 The charge densities in the transverse plane of the $u$ quark for the a unpolarized, $\mathbf{b}$ transversely polarized nucleon and $d$ quark for the $\mathbf{c}$ unpolarized, $\mathbf{d}$ transversely polarized nucleon. The polarization is along the $x$-direction

nucleon is taken along the $x$-axis i.e., $\phi_{s}=0$. The second term in Eq. (27) provides the deviation from circular symmetry of the unpolarized charge density [44]. We show the charge densities for the transversely polarized proton and neutron in Fig. 4a, b. The $u$ and $d$ quark charge densities for the transversely polarized nucleon are shown in Fig. 4c, d. Again, in Model I, the densities for proton and $u$ quark are in good agreement with the global parametrizations but deviate for the neutron and the $d$ quark. Only for the $d$ quark charge density, as shown in Fig. 4d, Model II agrees with the phenomenological parametrizations better than Model I. The comparison of the charge densities for the transversely polarized and unpolarized proton is shown in Fig. 5a and a similar plot for the neutron is shown in Fig. 5b. For the nucleons polarized along the $+x$ direction, the charge densities are shifted toward the negative $b_{y}$ direction. The deviation is much larger for the neutron compared to the proton.
The behavior is in agreement with the results reported in $[4,44,47]$.

We compare the up and down quark charge densities for the transversely polarized and unpolarized nucleon in Figs. $5 \mathrm{c}, \mathrm{d}$. The deviation or distortion from the symmetric unpolarized density is higher for the down quark than the up quark. The shifting of the charge density for the nucleons polarized in the $+x$ direction is towards the positive $b_{y}$ direction for the down quark but opposite for the up quark. In Fig. 6, we have shown a top view plot of the charge densities in the transverse plane for (a) an unpolarized proton, and (b) a proton polarized along $x$-direction in Model I. Similar plots for the neutron are shown in Fig. 6c, d. Due to the large anomalous magnetic moment, which produces an induced electric dipole moment in the $y$-direction, the distortion is higher in the case of a neutron [44]. The top view plots for the $u$ and $d$ quarks charge densities in the transverse plane for both 
unpolarized and transversely polarized nucleon are shown in Fig. 7a-d.

\section{Summary}

In this paper, we have presented a detailed study and comparison of the charge and anomalous magnetization densities for nucleons in the transverse plane in two models in AdS/QCD. We have also compared our results with the two standard phenomenological parametrizations of the form factors. Both the unpolarized and the transversely polarized nucleons have been considered in this work. The unpolarized densities are symmetric in the transverse plane, while for the transversely polarized nucleons they become distorted. If the nucleon is polarized along the $x$ direction, the densities get shifted toward the negative $y$-direction. We have also studied the flavor decompositions of the transverse densities, i.e., the charge and anomalous magnetization densities for individual $u$ and $d$ quark flavors. Our analysis shows that Model I reproduces the data much better than Model II. The agreement is not so good for the $d$ quark, which is consistent with the findings in [32], where the form factors for the $d$ quark were shown to deviate from the experimental results. For transversely polarized nucleon, the distortion in the $d$ quark charge density is found to be stronger than that for the $u$ quark and shifted in mutually opposite directions.

Acknowledgments We thank V. E. Lyubovitskij, S. J. Brodsky and G. F. de Teramond for insightful correspondence on form factors in AdS/QCD.

Open Access This article is distributed under the terms of the Creative Commons Attribution License which permits any use, distribution, and reproduction in any medium, provided the original author(s) and the source are credited.

Funded by $\mathrm{SCOAP}^{3}$ / License Version CC BY 4.0.

\section{References}

1. M. Burkardt, Phys. Rev D. 62, 071503 (2000)

2. M. Burkardt, Int. J. Mod. Phys. A 18, 173 (2003)

3. G.A. Miller, Phys. Rev. C 80, 045210 (2009)

4. G.A. Miler, Annu. Rev. Nucl. Part. Sci. 60, 1 (2010)

5. S. Venkat, J. Arrington, G.A. Miller, X. Zhan, Phys. Rev. C 83, 015203 (2011)

6. J. Maldacena, Adv. Theor. Math. Phys. 2, 231 (1998)

7. J. Polchinski, M.J. Strassler, Phys. Rev. Lett. 88, 031601 (2002)

8. J. Polchinski, M.J. Strassler, JHEP 0305, 012 (2003)

9. L. Cornalba, M.S. Costa, Phys. Rev. D 78, 096010 (2008)

10. R.C. Brower, M. Djuric, I. Sarcevic, C.-I. Tan, JHEP 1011, 051 (2010)
11. M.S. Costa, M. Djurić, Phys. Rev. D 86, 016009 (2012)

12. M.S. Costa, M. Djurić, N. Evans, JHEP 1309, 084 (2013)

13. S.J. Brodsky, G.F. de Téramond, Phys. Lett. B 582, 211 (2004)

14. S.J. Brodsky, G.F. de Téramond, Phys. Rev. Lett. 96, 201601 (2006)

15. G.F. de Téramond, S.J. Brodsky, Phys. Rev. Lett. 94, 201601 (2005)

16. J. Erlich, E. Katz, D.T. Son, M.A. Stephanov, Phys. Rev. Lett. 95, 261602 (2005)

17. T. Sakai, S. Sugimoto, Prog. Theo. Phys. 113, 843 (2005)

18. Z. Abidin, C.E. Carlson, Phys. Rev. D 79, 115003 (2009)

19. H. Forkel, M. Beyer, T. Frederico, Int. J. Mod. Phys. E 16, 2794 (2007)

20. W. de Paula, T. Frederico, H. Forkel, M. Beyer, Phys. Rev. D 79, 75019 (2009)

21. A. Vega, I. Schmidt, T. Branz, T. Gutsche, V.E. Lyubovitskij, Phys. Rev. D 80, 055014 (2009)

22. Z. Abidin, C.E. Carlson, Phys. Rev. D 77, 095007 (2008)

23. S.J. Brodsky, G.F. de Téramond, Phys. Rev. D 77, 056007 (2008)

24. S.J. Brodsky, G.F. de Téramond, Phys. Rev. D 78, 025032 (2008)

25. S.J. Brodsky, F. Cao, G.F. de Téramond, Phys. Rev. D 84, 075012 (2011)

26. G.F. de Téramond, H.G. Dosch, S.J. Brodsky, Phys. Rev. D 87, 075005 (2013)

27. S.J. Brodsky, G.F. de Téramond. arXiv:1203.4025 [hep-ph]

28. A. Vega, I. Schimdt, T. Gutsche, V.E. Lyubovitskij, Phys. Rev. D 83, 036001 (2011)

29. A. Vega, I. Schimdt, T. Gutsche, V.E. Lyubovitskij, Phys. Rev. D 85, 096004 (2012)

30. T. Gutsche, V.E. Lyubovitskij, I. Schmidt, A. Vega, Phys. Rev. D 86, 036007 (2012)

31. D. Chakrabarti, C. Mondal, Phys. Rev. D 88, 073006 (2013)

32. D. Chakrabarti, C. Mondal, Eur. Phys. J. C 73, 2671 (2013)

33. K. Hashimoto, T. Sakai, S. Sugimoto, Prog. Theor. Phys. 120, 1093 (2008)

34. J.R. Forshaw, R. Sandapen, Phys. Rev. Lett. 109, 081601 (2012)

35. M. Ahmady, R. Sandapen, Phys. Rev. D 87, 054013 (2013)

36. M. Ahmady, R. Sandapen, Phys. Rev. D 88, 014042 (2013)

37. Z. Abidin, C.E. Carlson, Phys. Rev. D 80, 115010 (2009)

38. M. Ahmady, R. Campbell, S. Lord, R. Sandapen (2014). arXiv:1401.6707 [hep-ph]

39. Z. Abidin, C.E. Carlson, Phys. Rev. D 78, 071502 (2008)

40. H.C. Ahn, D.K. Hong, C. Park, S. Siwach, Phys. Rev D. 80, 054001 (2009)

41. Z. Li, B.Q. Ma (2013). arXiv:1312.3451 [hep-ph]

42. G.A. Miller, Phys. Rev. Lett. 99, 112001 (2007)

43. O.V. Selyugin, O.V. Teryaev, Phys. Rev. D 79, 033003 (2009)

44. C.E. Carlson, M. Vanderhaeghen, Phys. Rev. Lett. 100, 032004 (2008)

45. M. Vanderhaeghen, T. Walcher. arXiv:1008.4225 [hep-ph]

46. C. Granados, C. Weiss, JHEP 1401, 092 (2014)

47. A. Silva, D. Urbano, H. Kim. arXiv:1305.6373 [hep-ph]

48. J.J. Kelly, Phys. Rev. C 66, 065203 (2002)

49. J.O. Gonzalez-Hernandez, S. Liuti, G.R. Goldstein, K. Kathuria, Phys. Rev. C 88, 065206 (2013)

50. J.J. Kelly, Phys. Rev. C 70, 068202 (2004)

51. R. Bradford, A. Bodek, H. Budd, J. Arrington, and. Nucl. Phys. Proc. Suppl. 159, 127 (2006)

52. S.J. Brodsky, G.F. de Téramond, H.G. Dosch. arXiv:1302.5399 [hep-ph]

53. H.R. Grigoryan, A.V. Radyushkin, Phys. Rev. D 76, 095007 (2007)

54. S.J. Brodsky, S.D. Drell, Phys. Rev. D 22, 2236 (1980)

55. G.D. Cates, C.W. de Jager, S. Riodian, B. Wojtsekhowski, Phys. Rev. Lett. 106, 252003 (2011) 